\title{
Análisis de la evolución de la concentración geográfica de los establecimientos de turismo rural en Cataluña
}

\author{
José Ma PRAT ForGA \\ Departamento de Geografía, Grupo de Investigación TUDISTAR \\ Universidad Autónoma de Barcelona \\ Josepmaria.prat@uab.cat \\ Gemma CÀnOVES VALIENTE \\ Departamento de Geografía, Grupo de Investigación TUDISTAR \\ Universidad Autónoma de Barcelona \\ gemma.canoves@uab.cat
}

Recibido: 13 de noviembre de 2013

Enviado a evaluar: 9 de enero de 2014

Aceptado: 30 de abril de 2014

\section{RESUMEN}

Para el estudio de la concentración industrial se han utilizado diversas técnicas cuantitativas. Sin embargo, no existen trabajos que analicen la concentración geográfica de los diversos tipos de establecimientos de turismo rural y su evolución temporal. El objetivo de este trabajo, por tanto, es analizar la concentración espacial de las diferentes tipologías de estos alojamientos en Cataluña, a nivel municipal, comarcal y de marca turística; y su evolución durante los años 2000, 2006 y 2012. Para ello, a partir de los datos estadísticos oficiales, se han calculado los índices de Gini, Ellison-Glaeser y Concentración Relativa. Los resultados obtenidos muestran una concentración territorial muy leve en estos años, aunque con un aumento de la oferta de estos alojamientos en un mayor número de municipios dentro de una misma comarca. Además, se percibe también la presencia de pequeñas aglomeraciones territoriales en el caso de las casas de pueblo compartidas.

Palabras clav e: Aglomeración geográfica, concentración espacial, análisis espacial, turismo rural, Cataluña, España.

\section{Analysis of the evolution of the geographic concentration of the establishments of rural tourism in Catalonia}

\begin{abstract}
For the study of the industrial concentration have used diverse quantitative technicians. However, they do not exist works analysing the geographic concentration of the diverse types of establishments of rural tourism and his temporary evolution. The aim of this work, therefore, is to analyse the space concentration of the different typologies of these accommodations in Catalonia, to municipal level, local and of tourist mark; and his evolution during the years 2000, 2006 and 2012. For this, from the official
\end{abstract}


statistical data, we are calculated the indexes of Gini, Ellison-Glaeser and Relative Concentration. The results obtained show a territorial concentration very slight in all these years, although increasing the offer of these accommodations in a greater number of municipalities inside a same region. Besides, it perceives the presence of small territorial agglomerations in the case of the houses of village shared.

Key words : Geographic agglomeration, space concentration, space analysis, rural tourism, Catalonia, Spain

\section{Analyse de l'évolution de la concentration géographique des établissements de tourisme rural en Catalogne}

\section{RÉSUMÉ}

Pour l'étude de la concentration industrielle ils se sont utilisé diverses techniciennes quantitatives. Pourtant, ils n'existent pas des travaux en analysant la concentration géographique des divers établissements de tourisme rural et son évolution temporelle. Le but de ce travail, par tellement, est analyser la concentration spatiale des logements en Catalogne, à niveau municipal, régional et de marque touristique; et son évolution pendant les ans 2000, 2006 et 2012. Pour cela, à partir des données statistiques officielles, ils s'appliquent les indices de Gini, Ellison-Glaeser et Concentration Relative. Les résultats obtenus montrent une concentration territoriale très légère en tous ces ans, bien que en augmentant l'offre de ces logements dans un majeur nombre de communes dedans d'une même région. En plus, il se perçoit la présence de petites agglomérations territoriales dans le cas des maisons de village partagées.

Mots c lés: Agglomération géographique, concentration spatiale, analyse spatiale, tourisme rural, la Catalogne, l'Espagne.

\section{INTRODUCCIÓN}

En la actualidad nos encontramos en un contexto caracterizado, entre otras cosas, por la globalización y las nuevas tecnologías, la crisis económica, la desintermediación y los nuevos hábitos de la demanda turística, donde la experiencia del turista, la competitividad entre destinos, la calidad de la oferta, la propuesta de actividades y la personalización son elementos clave del viaje (Ávila y Barrado, 2005). Ante esta situación se ha producido una evolución en la oferta de productos turísticos y el turismo rural no ha sido ajeno a este fenómeno. Se trata de un tipo de turismo considerado como "aquella actividad turística realizada en el espacio rural, compuesta por una oferta integrada de ocio, dirigida a una demanda motivada por el contacto con el entorno autóctono y que tenga una interrelación con la sociedad local", según la definición dada por la Comisión de las Comunidades Europeas (en Cànoves et al., 2006).

Por ello, es interesante avanzar en el conocimiento de los elementos que explican la evolución de la distribución territorial de esta oferta, para que en el futuro se puedan diseñar unas políticas públicas que favorezcan esta localización en las áreas más adecuadas, ya que las externalidades generadas por la concentración de estos alojamientos pueden ser una importante fuente de competitividad (Tickell, 2002), y 
las economías de aglomeración en el sector servicios no han estado especialmente estudiadas (Guimaraes et al., 2000). En este sector, trabajos recientes muestran que los servicios tienen sus propias estrategias de localización y el estudio de las aglomeraciones está ganando importancia (Capone y Boix, 2008).

El objetivo del presente trabajo es realizar un estudio cuantitativo sobre los niveles de concentración espacial de los establecimientos de turismo rural en Cataluña, analizando detalladamente su evolución en tres años: 2000, 2006 y 2012. Dicho análisis también se realiza para cada una de las tipologías existentes, teniendo en cuenta la clasificación establecida por el Instituto de Estadística de Cataluña (Idescat): casas de pueblo independientes, masías y masonerías (que se diferencian entre sí por ser la residencia de los dueños o de los masoveros de la finca) y casas de pueblo compartidas (que pueden alquilarse por habitaciones).

La hipótesis que aquí se plantea es que las economías de aglomeración juegan un papel activo en la localización geográfica de estos establecimientos. Para verificarla se cuantifican y valoran los niveles de concentración según las diferentes tipologías, la unidad territorial de análisis y los años seleccionados; comprobando si existen aglomeraciones y si éstas se reflejan en una localización concreta.

Para el estudio de la concentración geográfica de los diferentes sectores tradicionalmente se ha utilizado el índice de Gini, mientras que la especialización de los territorios se suele medir mediante el índice de Concentración Relativa (Strange, 2005; Capone y Boix, 2008). Además, la aparición en estos últimos años de indicadores de aglomeración, como el de Ellison-Glaeser, permiten controlar la influencia del tamaño de los establecimientos de turismo rural (número de plazas disponibles) en la localización de la oferta (Strange, 2005).

Así pues, en este artículo, después de esta breve introducción, se presenta una síntesis teórica sobre el turismo rural y su evolución en Cataluña, y sobre los conceptos de concentración y aglomeración geográfica. A continuación, se explica la metodología aquí utilizada y se analizan los resultados obtenidos. Finalmente, se comprueba la hipótesis propuesta, se exponen las conclusiones alcanzadas y se relaciona la bibliografía referenciada.

\section{SITUACIÓN DEL TURISMO RURAL EN CATALUÑA}

La necesidad de redescubrir el mundo rural por parte de los habitantes de las grandes ciudades es un factor que ayuda a entender el desarrollo del turismo rural (Pulido, 2008), el cual ha experimentado un fuerte crecimiento en Cataluña en los últimos años (Figuras 1 y 2). 
Figura 1. Evolución de la oferta de establecimientos de turismo rural en Cataluña.

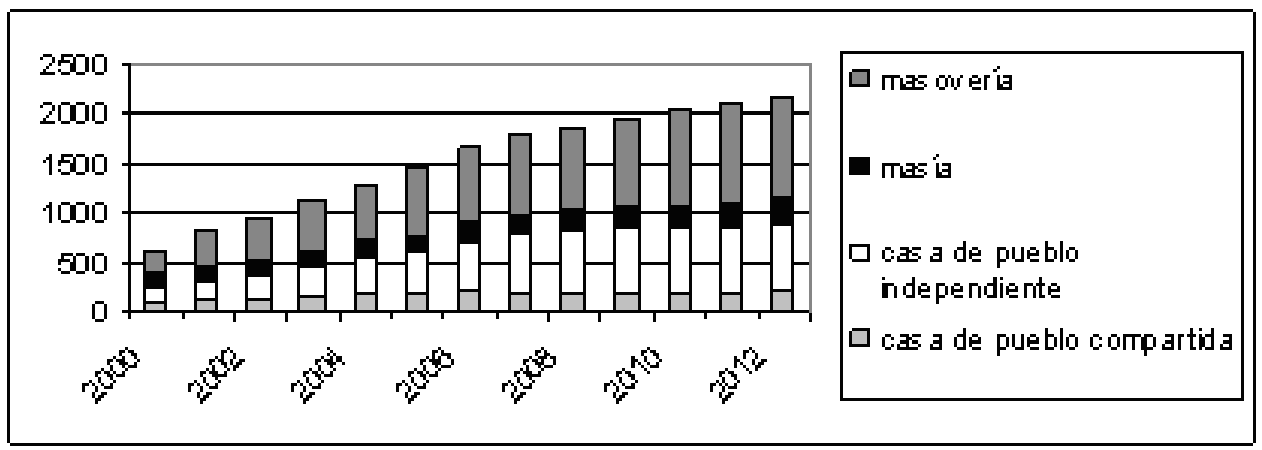

Fuente: Elaboración propia, a partir de Idescat.

Figura 2. Evolución de la oferta de plazas de turismo rural en Cataluña.

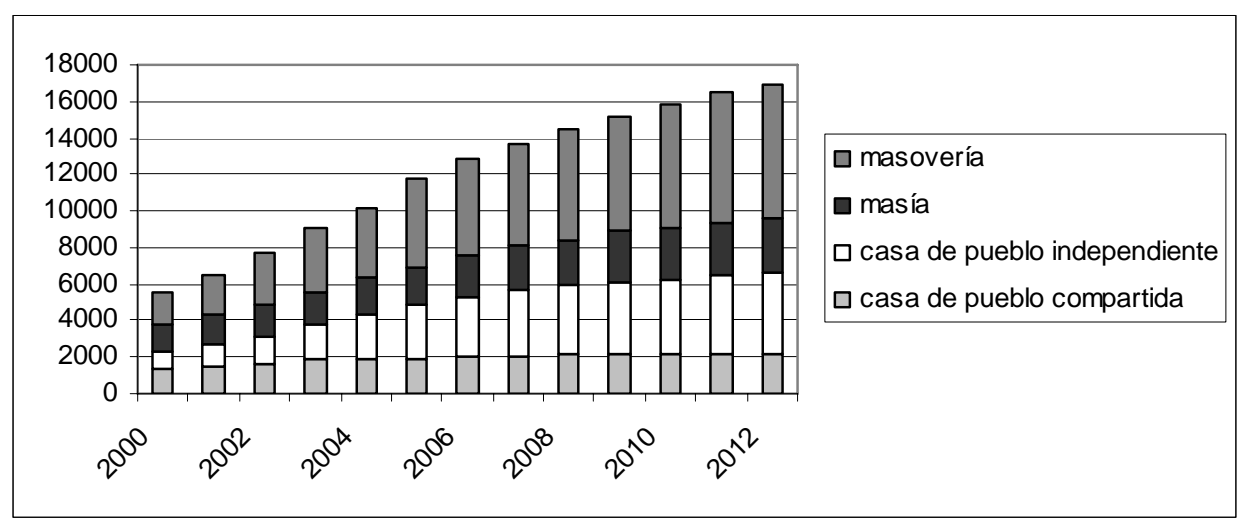

Fuente: Elaboración propia, a partir de Idescat.

Así, según los datos facilitados por el Idescat, en el año 2012 había 2.156 establecimientos de turismo rural, con 16.968 plazas. Seis años antes, en el 2006, la oferta era de 1.669 establecimientos y 12.865 plazas; y en el 2000 era de 645 establecimientos y 5.558 plazas. Por tanto, en estos doce años el número de estos establecimientos ha aumentado un $234 \%$ y el de plazas un $205 \%$, especialmente en el caso de las masoverías y de las casas de pueblo independientes. La mayoría de turistas que consumen estos productos residen en la propia Cataluña (el $88,8 \%$ en el 2011), frente al 4,6\% que procede del resto de España y el 6,6\% del extranjero. En cuanto al número de pernoctaciones, se ha pasado de 680.000 (año 2004) a 929.000 (año 2011). Se trata de una oferta con gran competencia interior, bajo grado de ocupación (108 días al año) y gran estacionalidad (en el primer trimestre la ocupación es del 13\%-19\%; en el segundo del $17 \%-23 \%$; en el tercero del $42 \%-44 \%$; y en el cuarto del $20 \%-24 \%$, Idescat, 2013). 
Este turismo ha permitido un aumento del consumo de otros productos y servicios (restauración, comercios, etc.), ha ayudado a frenar el despoblamiento en las zonas más favorecidas y, en algunos casos, está propiciando la llegada de nuevos pobladores, ha revalorizado los territorios de interior y a fortalecido la autoestima de la comunidad local (Cànoves et al., 2006). Es el resultado de una sociedad inmersa en un proceso de intensa urbanización, con una mayor sensibilidad medioambiental, una necesidad de retorno a las raíces rurales, una extensión y segmentación del tiempo de ocio $\mathrm{y}$, en definitiva, una nueva mirada hacia el espacio rural como lugar con patrimonio, cultura, paisaje y nuevas oportunidades (Cànoves et al., 2004). Una tendencia bastante común en estos últimos años es que los clientes se han ido inclinando hacia los alojamientos rurales independientes y las masoverías, tendiendo hacia un turismo rural alejado del agroturismo, prefiriendo no convivir con los propietarios de los establecimientos ni disfrutando de las actividades agrarias (Cànoves et al., 2006).

Territorialmente, la oferta está repartida poco uniformemente por todo el territorio catalán (Figura 3), ya que está concentrada en las comarcas más septentrionales (Pirineos y Pre-Pirineos), exceptuando el Valle de Arán y la Cerdanya, donde predomina el turismo residencial, mientras que las comarcas con menos oferta están en el litoral y el oeste.

Figura 3. Mapa de situación comarcal de los establecimientos de turismo rural en Cataluña (2012).

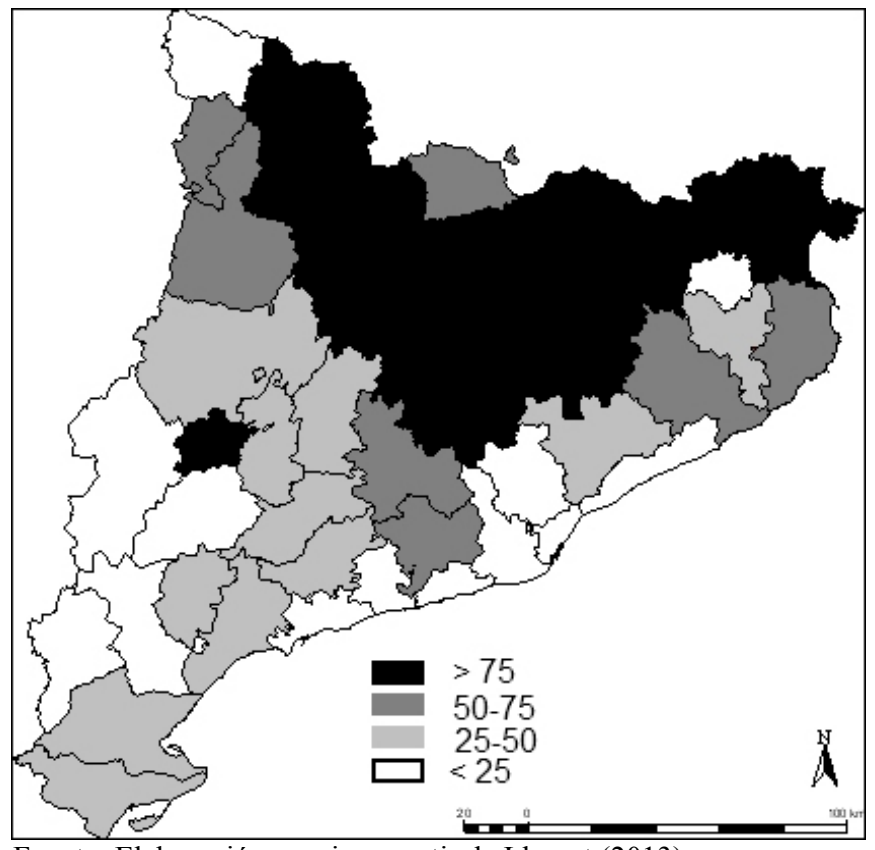

Fuente: Elaboración propia, a partir de Idescat (2013) 


\section{CONCENTRACIÓN GEOGRÁFICA Y AGLOMERACIÓN}

El concepto de aglomeración surge de la necesidad de distinguir entre la concentración geográfica de los establecimientos del mismo sector que están situados cerca unos de otros, de la concentración causada por la propia estructura sectorial (Alañon et al., 2007). La evidencia empírica indica que el proceso de localización no es aleatorio entre territorios, sino que se observan ciertas pautas generalizadas de localización, con una existencia simultánea de dos factores: aglomeración y externalidades (Ottaviano y Thisse, 2005). Es conveniente precisar que con las externalidades se ayuda a explicar la formación de las aglomeraciones, ya que muchos de los patrones habituales no eran explicados por la dotación de recursos naturales, ni por la existencia de economías de escala internas del sector (Devereux et al., 2004). Algunos ejemplos son las características propias del territorio (como sus recursos naturales) y el grado de movilidad del capital humano (Brenner, 2006).

Desde principios de la década de los 90's ha tenido lugar un renacimiento del interés de la literatura económica para analizar la localización de los determinantes de la actividad productiva y sus economías externas o de aglomeración (Krugman, 1991; Ybarra, 1991; Costa, 1992; Ellison y Glaeser, 1997, 1999; Viladecans, 2000, 2004; Giner y Santa María, 2002; Rosenthal y Strange, 2003; O'Donoghue y Gleave. 2004; Santa María, Giner y Fuster, 2004; Brenner, 2006; Viladecans y Jofre, 2006; Boix y Galletto, 2009). Este análisis, básicamente empresarial, se ha empezado a usar desde una perspectiva geográfica en estos últimos años, utilizando nuevas metodologías econométricas que incorporan el espacio geográfico en sus cálculos y apareciendo nuevos modelos teóricos. Sin embargo, dicho análisis, eminentemente empírico, aunque ha estado ampliamente estudiado a través de la teoría de la localización, no ha obtenido un consenso que permita determinar cual es el índice de medición más adecuado para su estudio (Greene, 2003; Viladecans, 2004).

En el caso del turismo, estos análisis espaciales aún son muy minoritarios, por lo que se mantiene la disyuntiva de cual es el mejor índice a utilizar, ya que a pesar de que las interacciones entre la industria turística y el territorio han estado estudiadas, "el éxito de una firma turística no depende sólo de su estrategia y posicionamiento, sino de la forma en que consiga integrarse en su ambiente" (Vanhove, 2002). Por su parte, Millán (2008) resalta la importancia de promover la diversificación de las actividades turísticas para aprovechar las potencialidades del territorio.

Así, siguiendo las pautas marcadas por los estudios econométricos empresariales (entre otros, Duranton y Overman, 2005; Brenner, 2006; Soler, 2006; Viladecans y Jofre, 2006; Capone y Boix, 2008), en este trabajo se analiza la concentración geográfica y la posible aglomeración de los diferentes tipos de establecimientos de turismo rural. Para ello se calculan tres índices: el índice de Gini, el índice de Concentración Relativa y el índice de Ellison-Glaeser, que corrige las limitaciones metodológicas de los anteriores, ya que aquellos no tienen en cuenta la incidencia del tamaño de los establecimientos (De Lucio, 1998; Viladecans y Jofre, 2006). Ahora bien, también es necesario destacar las diferencias obtenidas en los resultados cuando se calculan estos índices en diferentes entornos territoriales (Krugman, 1991) y, por lo 
tanto, la unidad de análisis a elegir aparece como un factor clave en el estudio de la dinámica de la concentración del sector.

\section{METODOLOGÍA}

La base de datos utilizada para llevar a cabo este análisis descriptivo es la territorial del Idescat sobre el turismo rural (número de establecimientos y de plazas, para cada tipología, en los años 2000- 2006-2012), a nivel comarcal (Figura 5) y municipal, con el objetivo de analizar la evolución de las diferentes variables (tipologías y unidades territoriales). Esos datos y análisis también se han agregado según las marcas turísticas decretadas por la Generalitat de Cataluña (Figura 6).

\section{1. ÍNDICE DE GINI}

Este índice informa sobre la mayor o menor disparidad con que una determinada variable se distribuye entre los individuos que forman la población de interés (Greene, 2003). En este trabajo, el índice de Gini analiza la distribución geográfica de los diferentes tipos de establecimientos de turismo rural entre las tres unidades territoriales seleccionadas (municipal, comarcal y marca turística), tomando valores entre cero (distribución totalmente igualitaria) y uno (totalmente concentrada).

Como que en Cataluña no se dispone de estadísticas oficiales sobre el número de plazas en cada alojamiento de turismo rural, en este trabajo se ha tenido que aproximar el índice de Gini por intervalos de tamaño, mediante tramos de diez establecimientos para el caso del análisis comarcal, tres para el municipal y cincuenta para el análisis por marcas turísticas. Para ello, la fórmula utilizada (Gini, 1912, en Greene, 2003) es la siguiente:

con:

$$
\mathrm{IG}=\left(\sum\left(\mathrm{p}_{\mathrm{k}}-\mathrm{q}_{\mathrm{k}}\right)\right) /\left(\sum_{\mathrm{k}=1} \mathrm{p}_{\mathrm{k}}\right)_{\mathrm{k}=1}^{\mathrm{n}}
$$

$\mathrm{n}$

$$
\begin{gathered}
\mathrm{p}_{\mathrm{i}}=\mathrm{F}_{\mathrm{k}} /\left(\sum_{\mathrm{k}=1} \mathrm{~F}_{\mathrm{k}}\right) \\
\mathrm{n} \mathrm{q}_{\mathrm{i}}=\left(\mathrm{f}_{\mathrm{k}} * \mathrm{~F}_{\mathrm{k}}\right) /\left(\sum_{\mathrm{k}} * \mathrm{~F}_{\mathrm{k}}\right)_{\mathrm{k}=1}
\end{gathered}
$$

siendo: $\quad k$ el número de tramos (de 1 a $n$ ) en cada tipología $i$

$f_{k}$ la frecuencia de establecimientos de la tipología $i$ en el tramo $k$

$F_{k}$ la frecuencia acumulada de $f_{k}$ 


\section{2. ÍNDICE DE CONCENTRACIÓN RELATIVA}

El índice de Concentración Relativa (CR) mide la importancia de las unidades territoriales donde hay más establecimientos de cada tipología (Rosenthal y Strange, 2003; Viladecans, 2004; Strange, 2005). El índice CR-3 mide el porcentaje de estos alojamientos que están acumulados en los tres municipios, comarcas y marcas turísticas con mayor cantidad de ellos. El índice CR-5, hace lo propio con los cinco primeros y el CR-10 con los diez primeros. En el caso presente, como que en Cataluña sólo hay nueve marcas turísticas (Figura 6), se han calculado los índices CR-3 y CR-5, mientras que para las comarcas (Figura 5) y municipios, los índices seleccionados han sido CR-3 y CR-10. En todos los casos, la fórmula para el cálculo de estos índices es:

$$
\mathrm{CR}-\mathrm{m}=\sum_{\mathrm{j}=1}^{\mathrm{m}}\left(\mathrm{L}_{\mathrm{ij}} / \mathrm{L}_{\mathrm{i}}\right)
$$

siendo: $i$ el tipo de establecimiento de turismo rural analizado

$j$ la localidad analizada (municipio/comarca/marca turística)

$m$ el $\mathrm{n}^{\mathrm{o}}$ de posiciones analizadas (3, 5 ó 10$)$

$L_{i j}$ el $\mathrm{n}^{\mathrm{o}}$ de establecimientos de turismo rural de la tipología $i$ presentes en la localidad analizada

$L_{i}$ el $n^{\circ}$ total de este tipo de establecimientos de turismo rural en Cataluña

\section{3. ÍNDICE DE CONCENTRACIÓN GEOGRÁFICA DE ELLISON-GLAESER (IEG).}

Este índice, además de analizar la concentración geográfica de un determinado elemento, intenta averiguar que parte de esta concentración se debe a las fuerzas de aglomeración que llevan a estos establecimientos a localizarse en una misma ubicación geográfica o si influyen otros elementos, como el azar (Ellison y Glaser, 1999; Strange, 2005). De este modo, para conocer si la distribución espacial es realizada aleatoriamente por todo el territorio, es necesario ver si la concentración se debe a que solamente hay unos cuantos establecimientos de este tipo en este territorio. Para ello se utiliza una medida que nos aproxime a sus características no espaciales (Strange, 2006). Ellison y Glaeser (1999) recurren al índice de Hirschman-Herfindhal $(\mathrm{HH})$, que aproxima la concentración de mercado y es muy utilizado en el campo de la organización industrial (Viladecans, 2004).

Además, aunque los índices de Concentración Relativa y de Gini ya permiten ver algunas diferencias en la distribución territorial entre las diferentes tipologías, ninguno de ambos tiene en cuenta la dimensión de su capacidad de alojamiento, ya que las plazas pueden estar desigualmente distribuidas. Por esta razón, Krugman (1991) y Audretsch y Feldman (1996) propusieron el índice de Gini Espacial (IGE), donde la máxima igualdad se da cuando la distribución geográfica de una de las tipologías de estos establecimientos es exactamente la misma que la global. 
Al igual que en el caso del índice de Gini, aquí también se ha aproximado el índice de Hirschman-Herfindhal por los mismos intervalos de tamaño que antes. Las fórmulas utilizadas para calcular estos índices son:

con:

$$
\begin{gathered}
\mathrm{IEG}=(\mathrm{IGE}-\mathrm{HH}) /(1-\mathrm{HH}) \\
\mathrm{HH}=\sum_{\mathrm{k}=1}^{\mathrm{n}}\left(\left(\mathrm{L}_{\mathrm{ik}} / \sum_{\mathrm{k}=1}^{\mathrm{n}} \mathrm{L}_{\mathrm{ik}}\right)^{2} \cdot\left(1 / \mathrm{N}_{\mathrm{ik}}\right)\right) \\
\mathrm{n} \operatorname{n}=\sum\left(\left(\mathrm{s}_{\mathrm{ik}}-\mathrm{x}_{\mathrm{ik}}\right)^{2}\right) /\left(1 / \sum \mathrm{x}_{\mathrm{ik}}{ }^{2}\right) \quad \mathrm{n} \\
\mathrm{k}=1
\end{gathered}
$$

siendo: $i$ el tipo de establecimiento de turismo rural analizado $j$ la localidad analizada (municipio/comarca/marca turística) $k$ el $n^{\circ}$ de tramos para cada localidad analizada (de 1 a $n$ ) $L_{i k}$ el $\mathrm{n}^{\mathrm{o}}$ de plazas de turismo rural de la tipología $i$ presentes en el tramo analizado

$N_{i k}$ el $n^{\circ}$ de establecimientos de la tipología $i$ en cada tramo $k$ $\mathrm{s}_{\mathrm{ik}}$ el porcentaje de plazas de la tipología $i$ en cada tramo $k$ con respecto al total de plazas de esta tipología en Cataluña

$\mathrm{x}_{\mathrm{ik}}$ el porcentaje de plazas totales de turismo rural en cada tramo $k$ con respecto al total de plazas en Cataluña

El índice de Ellison-Glaeser (IEG) puede tomar tres valores cualitativamente diferentes. Los valores negativos denotan que la localización de los establecimientos de una determinada tipología está menos concentrada que el total de las plazas ofertadas, teniendo en cuenta el efecto del número de establecimientos de turismo rural existentes en cada categoría. Así, un valor próximo a cero indica una aglomeración próxima a la del global de la tipología analizada, mientras que un valor positivo pone de relieve la existencia de fuerzas de aglomeración que llevan a estos alojamientos a localizarse en unos determinados lugares y no en otros (Viladecans y Jofre, 2006). En nuestro caso, siguiendo los criterios de Ellison-Glaeser, consideramos una tipología como poco concentrada cuando IEG $<0,02$, moderadamente concentrada si $0,02<\mathrm{IEG}<0,05$ y muy concentrada si IEG $>0,05$. 


\section{RESULTADOS DEL ANÁLISIS}

\section{1. ÍNDICE DE GINI}

Los resultados de los cálculos del índice de Gini para cada una de las tres tipologías de establecimientos de turismo rural, en cada uno de los tres años aquí considerados, son los siguientes (Tabla 1):

Tabla 1. Resultados del Índice de Gini.

\begin{tabular}{|l|c|c|c|c|c|}
\hline & & $\begin{array}{c}\text { casa de pueblo } \\
\text { compartida }\end{array}$ & $\begin{array}{c}\text { casa de pueblo } \\
\text { independiente }\end{array}$ & $\begin{array}{c}\text { masía / } \\
\text { masoveria total }\end{array}$ \\
\hline comarcas & 2012 & 0,2755 & 0,3047 & 0,4193 & 0,3274 \\
& 2006 & 0,2243 & 0,2839 & 0,3025 & 0,3261 \\
& 2000 & 0,0978 & 0,3507 & 0,1968 & 0,2487 \\
\hline municipios & & & & & \\
& 2012 & 0,0741 & 0,0785 & 0,1234 & 0,0842 \\
& 2006 & 0,0768 & 0,0841 & 0,0342 & 0,0824 \\
& 2000 & 0,0168 & 0,0879 & 0,1011 & 0,0853 \\
\hline marcas turísticas & & & & & \\
& 2012 & 0,1848 & 0,3736 & 0,3077 & 0,390 \\
& 2006 & 0,3120 & 0,3825 & 0,3120 & 0,415 \\
& 2000 & 1,0000 & 0,3575 & 0,1848 & 0,487 \\
\hline
\end{tabular}

Fuente: Elaboración propia

Tal como se puede observar en la tabla anterior, a nivel municipal los valores obtenidos son bastante bajos y, en ocasiones, muy próximos a cero; lo que demuestra que la dispersión geográfica de estos establecimientos es muy alta. En cuanto a su evolución en el tiempo, las casas de pueblo independientes han mantenido un índice alrededor de 0,08 (una alta dispersión territorial), con un decremento del 10,69\% entre 2000 y 2012; mientras que en las casas de pueblo compartidas dicho índice ha pasado de 0,0168 a 0,0741 , lo que representa un incremento de $341,07 \%$ (especialmente destacable en sus seis primeros años, donde el aumento fue del $357,14 \%$ ), aunque se mantiene una dispersión geográfica muy alta a nivel municipal. En cuanto a las masías y masoverías, a pesar de que en este período se ha producido un incremento del $22,06 \%$, se mantiene la alta dispersión (con valores alrededor de $0,1)$, destacando un aumento del $260,82 \%$ en los últimos seis años, que ha contrarrestado la bajada del $66,17 \%$ en los seis primeros años.

A nivel comarcal los resultados obtenidos son algo más elevados, pasando del 0,27 (en el 2000) al 0,42 (en el 2012), pero con diferencias apreciables según la tipología. 
Así, en el caso de las casas de pueblo compartidas se ha producido un incremento del $181,70 \%$, especialmente destacable en los seis primeros años (donde el aumento fue del 129,35\%). En cuanto a las casas de pueblo independientes, los valores han oscilado alrededor del 0,3 ; lo que manifiesta una dispersión geográfica menos acusada y una cierta estabilidad territorial. Finalmente, para las masías y masoverías el índice ha dado unos resultados mayores con el tiempo, pasando del 0,1968 (año 2000) al 0,3025 (año 2006) y al 0,4193 (año 2012); lo que representa una reducción en la dispersión geográfica de estos establecimientos, aumentando la concentración en el 113,06\%.

En el caso de las marcas turísticas, las casas de pueblo independientes tienen una concentración relativamente baja (siempre con valores inferiores a 0,4) y un incremento del $4,5 \%$ a lo largo del periodo. Por su parte, las masías y masoverías han experimentado un aumento del 66,5\%, especialmente en los primeros seis años $(68,83 \%)$. En cuanto a las casas de pueblo compartidas, han sufrido una fuerte dispersión, ya que su concentración territorial ha disminuido un $81,52 \%$, pasando de una gran concentración en pocas marcas turísticas en el 2000, con un índice cercano a uno (Pirineus, Costa Brava y Catalunya Central), a una gran dispersión geográfica en el 2012, con un valor de 0,1848 .

En el conjunto de las tres tipologías se observa que en estos doce años se ha producido una evolución en la distribución geográfica de estos establecimientos, de manera que la oferta se ha extendido a más marcas turísticas, aunque se ha redistribuido comarcalmente, concentrándose más alojamientos en determinadas comarcas.

\section{2. ÍNDICE DE CONCENTRACIÓN RELATIVA}

En las tablas siguientes (Tablas 2 a 10) se presentan los resultados para los tres años, donde, al ser valores porcentuales, un $\mathrm{CR}=1$ indica que el $100 \%$ de los establecimientos del tipo analizado están concentrados en la misma localidad. Así, analizando los resultados por comarcas (Tablas 2 a 4), se puede observar que en las casas de pueblo independientes la mayor oferta en el año 2012 estaba en el Pallars Sobirà y en el Alt Empordà (ambas con un 9\% del total), mientras que en el 2006 la lista la encabezaba Osona (con un 8\%) y en el 2000 lo hacían la Alta Ribagorça (con un $9 \%$ ), la Garrotxa y el Berguedà (ambas con un $8 \%$ ). 
Tabla 2. Resultados del Índice de Concentración Relativa a nivel comarcal (año 2012).

\begin{tabular}{|cc|cc|cc|cc|}
\hline $\begin{array}{c}\text { casa de pueblo } \\
\text { independiente }\end{array}$ & & $\begin{array}{c}\text { casa de pueblo } \\
\text { compartida }\end{array}$ & $\mathbf{m}$ & asía/masoveria & & \multicolumn{2}{c|}{ total } \\
\hline Pallars Sobirà & 0,09 & Pallars Sobirà & 0,13 & Baix Empordà & 0,10 & Alt Empordà & 0,07 \\
Alt Empordà & 0,09 & Alta Ribagorça & 0,11 & Garrotxa & 0,09 & Berguedà & 0,06 \\
Alt Urgell & 0,07 & Pallars Jussà & 0,10 & Osona & 0,09 & Osona & 0,06 \\
Alta Ribagorça & 0,07 & Alt Empordà & 0,07 & Ripollès & 0,07 & Garrotxa & 0,06 \\
Pallars Jussà & 0,05 & Cerdanya & 0,06 & Solsonès & 0,07 & Ripollès & 0,06 \\
Segarra & 0,05 & Priorat & 0,06 & Pla de l'Estany & 0,06 & Solsonès & 0,05 \\
Noguera & 0,04 & Alt Urgell & 0,05 & Alt Empordà & 0,06 & Pallars Sobirà & 0,05 \\
Priorat & 0,04 & Berguedà & 0,05 & Bages & 0,06 & Pla de l'Estany & 0,04 \\
Ripollès & 0,04 & Val d'Aran & 0,04 & Berguedà & 0,04 & Bages & 0,04 \\
Urgell & 0,04 & Baix Camp & 0,03 & Selva & 0,04 & Alt Urgell & 0,04 \\
\hline CR-3 0, & $\mathbf{2 5}$ & CR-3 & $\mathbf{0 , 3 5}$ & CR-3 & $\mathbf{0 , 2 8}$ & CR-3 & $\mathbf{0 , 1 9}$ \\
\hline CR-10 0, & $\mathbf{5 7}$ & CR-10 & $\mathbf{0 , 7 2}$ & CR-10 & $\mathbf{0 , 6 8}$ & CR-10 & $\mathbf{0 , 5 2}$ \\
\hline
\end{tabular}

Fuente: Elaboración propia.

Tabla 3. Resultados del Índice de Concentración Relativa a nivel comarcal (año 2006).

\begin{tabular}{|cc|cc|cc|c}
\hline $\begin{array}{c}\text { casa de pueblo } \\
\text { independiente }\end{array}$ & & $\begin{array}{c}\text { casa de pueblo } \\
\text { compartida }\end{array}$ & & $\begin{array}{c}\text { masía/ } \\
\text { masoveria }\end{array}$ & to & tal \\
\hline Osona & 0,08 & Pallars Sobirà & 0,17 & Garrotxa & 0,11 & Osona \\
Solsonès & 0,06 & Pallars Jussà & 0,11 & Ripollès & 0,09 & Alt Empordà \\
Berguedà & 0,06 & Alta Ribagorça & 0,11 & Berguedà & 0,09 & Berguedà \\
Alt Empordà & 0,06 & Alt Urgell & 0,06 & Alt Empordà & 0,09 & Garrotxa \\
Garrotxa & 0,06 & Alt Empordà & 0,06 & Baix Empordà & 0,06 & Pallars Sobirà \\
Ripollès & 0,05 & Cerdanya & 0,05 & Bages & 0,06 & Solsonès \\
Pla de l'Estany & 0,04 & Val d'Aran & 0,05 & Pla de l'Estany & 0,05 & Ripollès \\
Bages & 0,04 & Priorat & 0,05 & Osona & 0,05 & Alta Ribagorça \\
Pallars Sobirà & 0,04 & Noguera & 0,04 & Selva & 0,04 & Pla de l'Estany \\
Alt Urgell & 0,04 & Berguedà & 0,03 & Vallès Oriental & 0,04 & Alt Urgell \\
\hline CR-3 0, & $\mathbf{2 1}$ & $\mathbf{C R - 3 ~ 0}$ & $\mathbf{4 0}$ & $\mathbf{C R - 3}$ & $\mathbf{0 , 2 9}$ & CR-3 \\
\hline CR-10 0 & $\mathbf{5 3}$ & $\mathbf{C R - 1 0}$ & $\mathbf{0 , 7 5}$ & $\mathbf{C R - 1 0}$ & $\mathbf{0 , 6 7}$ & CR-10 \\
\hline
\end{tabular}

Fuente: Elaboración propia. 
Tabla 4. Resultados del Índice de Concentración Relativa a nivel comarcal (año 2000).

\begin{tabular}{|cc|cc|cc|c}
\hline $\begin{array}{c}\text { casa de pueblo } \\
\text { independiente }\end{array}$ & $\begin{array}{c}\text { casa de pueblo } \\
\text { compartida }\end{array}$ & $\begin{array}{c}\text { masía } \\
\text { /masoveria }\end{array}$ & total & \\
\hline Alta Ribagorça & 0,09 & Garrotxa & 0,14 & Pallars Sobirà & 0,20 & Alta Ribagorça \\
Garrotxa & 0,08 & Alt Empordà & 0,10 & Alta Ribagorça & 0,17 & $\begin{array}{c}\text { Pallars Sobirà } \\
\text { Berguedà }\end{array}$ \\
Osona & 0,08 & Ripollès & 0,08 & Pallars Jussà & 0,11 & Garrotxa \\
Solsonès & 0,06 & Bages & 0,07 & Alt Urgell & 0,08 & Alt Urgell \\
Alt Urgell & 0,05 & Selva & 0,07 & Cerdanya & 0,07 & Alt Empordà \\
Alt Empordà & 0,05 & Berguedà & 0,05 & Noguera & 0,05 & Osona \\
Pallars Sobirà & 0,05 & Osona & 0,05 & Montsià & 0,04 & Pallars Jussà \\
Ripollès & 0,04 & Pla de l'Estany & 0,05 & Baix Empordà & 0,03 & Ripollès \\
Bages & 0,04 & Vallès Oriental & 0,04 & Priorat & 0,03 & Bages \\
\hline CR-3 0,25 & & CR-3 & $\mathbf{0 , 3 2}$ & CR-3 & $\mathbf{0 , 4 7}$ & CR-3 \\
\hline CR-10 0,61 & & CR-10 & $\mathbf{0 , 7 4}$ & CR-10 & $\mathbf{0 , 8 6}$ & CR-10 \\
\hline
\end{tabular}

Fuente: Elaboración propia.

Como se puede apreciar en las tablas anteriores, entre las diez comarcas con mayor oferta de casas de pueblo independientes solamente había un 53\% (año 2006) y un 61\% (año 2000) de estos establecimientos, y entre las tres primeras el porcentaje era del 21\%-25\%; lo que demuestra su expansión territorial en Cataluña.

Sin embargo, este fenómeno varía en el caso de las casas de pueblo compartidas, cuya localización geográfica comarcal está algo más concentrada, ya que entre las diez con más alojamientos de este tipo se reparten el $72 \%-75 \%$ de la oferta, y entre las tres primeras el 32\%-40\%. En detalle, la Garrotxa, que en el 2000 ocupaba la primera posición (con un 14\% del total), en el 2012 ha pasado a tener menos del 4\%, mientras que, en cambio, el Pallars Sobirà, que tenía en el 2000 menos del 4\% ha subido hasta el 13\%-17\%. También destacan los casos de la Alta Ribagorça (con un $11 \%$, tanto en el 2006 como en el 2012) y el Pallars Jussà (con un 10\%-11\%), teniendo ambas comarcas en el 2000 menos del 4\%. Respecto a las masías y masoverías, se ha pasado de una gran concentración territorial (entre las diez primeras comarcas se ofertaba el $86 \%$ de este tipo de alojamientos en el 2000 , y el $47 \%$ entre las tres primeras) se ha pasado al $68 \%$ y al $28 \%$, respectivamente. Esta redistribución explica que el Pallars Sobirà (con el 20\% de estos establecimientos en el 2000) y la Alta Ribagorça (el 17\%), hayan dejado los puestos primeros de esta clasificación al Baix Empordà (con el 10\%), la Garrotxa y Osona (ambas con el 8\%), quedándose los dos líderes anteriores por debajo del $4 \%$, lo que representa un decremento de un $17 \%$.

En valores absolutos, la oferta conjunta de establecimientos de turismo rural ha aumentado considerablemente en algunas comarcas, mientras que en otras, con más madurez en este tipo de turismo, se ha mantenido más estabilizada. También se puede observar como, a nivel comarcal, el turismo rural no está reñido con el turismo de litoral, ya que el Alt Empordà (con la Costa Brava localizada en su litoral) ocupa la 
primera posición teniendo en cuenta todas las tipologías de establecimientos de turismo rural, con una oferta del $7 \%$ del total. De esta manera, se ha desarrollado un turismo rural que ha servido de complemento al turismo más tradicional, ya que en dicha comarca se ha pasado de una oferta de 34 establecimientos de turismo rural en el 2000 a 149 en el 2012, especialmente localizados en sus territorios de interior.

Seguidamente se analizan los resultados de este índice a nivel municipal para cada unos de los tres años aquí considerados, obteniéndose las tablas siguientes (Tablas 5 a 7):

Tabla 5. Resultados del Índice de concentración relativa a nivel municipal (año 2012).

\begin{tabular}{|c|c|c|c|c|c|c|c|}
\hline $\begin{array}{l}\text { casa de pueblo } \\
\text { independiente }\end{array}$ & & $\begin{array}{c}\text { casa de pueblo } \\
\text { compartida }\end{array}$ & & $\begin{array}{c}\text { masia } \\
/ \text { masoveria }\end{array}$ & total & & \\
\hline Vall de Boí & 0,05 & Vall de Boí & 0,06 & Vall de Bianya & & Vall de Boí & 0,02 \\
\hline Soriguera & 0,02 & Pont de Suert & 0,03 & Lladurs & 0,02 & Vall de Bianya & 0,01 \\
\hline Alins & 0,02 & Alins & 0,02 & Deltebre & 0,02 & Lladurs & 0,01 \\
\hline Torre de Cabdella & 0,02 & Alt Àneu & 0,02 & Casserres & 0,01 & Vilademuls & 0,01 \\
\hline Montferrer i Castellbò & 0,02 & Conca de Dalt & 0,02 & $\begin{array}{l}\text { Vilademuls } \\
\text { Sant Joan de les }\end{array}$ & 0,01 & Deltebre & 0,01 \\
\hline Lles de Cerdanya & 0,01 & Lles & 0,02 & Abadesses & 0,01 & Soriguera & 0,01 \\
\hline Pont de Suert & 0,01 & Rialp & 0,02 & Navès & 0,01 & Casserres & 0,01 \\
\hline Calonge de Segarra & 0,01 & Torre de Cabdella, la & 0,02 & Llosses, les & 0,02 & $\begin{array}{c}\text { Sant Joan de les } \\
\text { Abadesses }\end{array}$ & 0,01 \\
\hline Rialp & 0,01 & Valls de Valira, les & 0,02 & Odèn & 0,01 & Alins & 0,01 \\
\hline Pujalt & 0,01 & Vielha e Mijaran & 0,02 & Arbúcies & 0,01 & Pont de Suert & 0,01 \\
\hline CR-3 0 &, 09 & CR-3 & 0,12 & CR-3 & 0,05 & CR-3 & 0,05 \\
\hline CR-10 0 & ,19 & CR-10 & 0,26 & CR-10 & 0,14 & CR-10 & 0,11 \\
\hline
\end{tabular}

Fuente: Elaboración propia.

Tabla 6. Resultados del Índice de concentración relativa a nivel municipal (año 2006).

\begin{tabular}{|c|c|c|c|c|c|c|c|}
\hline $\begin{array}{c}\text { casa de pueblo } \\
\text { independiente }\end{array}$ & & $\begin{array}{c}\text { casa de pueblo } \\
\text { compartida }\end{array}$ & & $\begin{array}{c}\text { masia } \\
\text { /masoveria }\end{array}$ & total & & \\
\hline Vall de Boí & 0,03 & Vall de Boí & 0,07 & Vilademuls & 0,04 & Vall de Bo & 0,03 \\
\hline Lladurs & 0,01 & Vielha e Mijaran & 0,04 & Vall d'en Bas & 0,03 & Vilademuls & 0,01 \\
\hline Deltebre & 0,01 & Baix Pallars & 0,03 & Vall de Bianya & 0,03 & Vall de Bianya & 0,01 \\
\hline Vall de Bianya & 0,01 & Conca de Dalt & 0,02 & Llosses, les & 0,02 & Torre de Cabdella & 0,01 \\
\hline $\begin{array}{c}\text { Odèn } \\
\text { Sant Joan de les }\end{array}$ & 0,01 & Alins & 0,02 & Argelaguer & 0,02 & Deltebre & 0,01 \\
\hline Abadesses & 0,01 & Valls de Valira & 0,02 & Arbúcies & 0,02 & Lladurs & 0,01 \\
\hline Torre de Cabdella & 0,01 & Torre de Cabdella & 0,02 & Aiguamúrcia & 0,02 & Alins & 0,01 \\
\hline Casserres & 0,01 & Pont de Suert & 0,02 & Planoles & 0,02 & Casserres & 0,01 \\
\hline Alins & 0,01 & Vilaller & 0,02 & $\begin{array}{c}\text { Peralada } \\
\text { Cruîlles, Monells i }\end{array}$ & 0,02 & Soriguera & 0,01 \\
\hline Soriguera & 0,01 & Lles & 0,02 & Sant Sadurní & 0,02 & Odèn & 0,01 \\
\hline CR-3 0, & 06 & CR-3 & 0,14 & CR-3 & 0,09 & CR-3 & $\mathbf{0 , 0 6}$ \\
\hline CR-10 0, & 13 & CR-10 & $\mathbf{0 , 3 0}$ & CR-10 & $\mathbf{0 , 2 0}$ & CR-10 & $\mathbf{0 , 1 3}$ \\
\hline
\end{tabular}

Fuente: Elaboración propia. 
Tabla 7. Resultados del Índice de concentración relativa a nivel municipal (año 2000).

\begin{tabular}{|cc|cc|cc|cc}
\hline $\begin{array}{c}\text { casa de pueblo } \\
\text { independiente }\end{array}$ & \multicolumn{2}{|c|}{$\begin{array}{c}\text { casa de pueblo } \\
\text { compartida }\end{array}$} & $\begin{array}{c}\text { masía/ } \\
\text { masoveria }\end{array}$ & tot & al \\
\hline Vall de Boí & 0,09 & Vall de Bianya & 0,04 & Vall de Boí & 0,10 & Vall de Boí & 0,08 \\
Vall de Bianya & 0,03 & Vilademuls & 0,03 & Vielha e Mijaran & 0,06 & Vall de Bianya & 0,02 \\
Deltebre & 0,02 & Argelaguer & 0,03 & Alins & 0,04 & Vielha e Mijaran & 0,02 \\
Sagàs & 0,02 & Arbúcies & 0,03 & Vilaller & 0,03 & Alins & 0,02 \\
Sant Mateu de Bages & 0,02 & Sant Mateu de Bages & 0,03 & Pont de Suert & 0,03 & Valls de Valira & 0,02 \\
Valls de Valira & 0,02 & Santa Susanna & 0,02 & Conca de Dalt & 0,03 & Sant Mateu de Bages & 0,02 \\
Casserres & 0,02 & Vall d'en Bas & 0,02 & Valls de Valira & 0,03 & Torre de Cabdella & 0,01 \\
Lladurs & 0,02 & Beuda & 0,02 & Alt Àneu & 0,03 & Deltebre & 0,01 \\
Torre de Cabdella & 0,02 & Sant Ferriol & 0,02 & Lles & 0,03 & Sagàs & 0,01 \\
Alins & 0,01 & Siurana & 0,02 & Àger & 0,03 & Casserres & 0,01 \\
\hline CR-3 0 & $\mathbf{1 4}$ & CR-3 & $\mathbf{0 , 0 9}$ & CR-3 & $\mathbf{0 , 2 0}$ & CR-3 & $\mathbf{0 , 1 2}$ \\
\hline CR-10 0 & $\mathbf{2 6}$ & CR-10 & $\mathbf{0 , 2 3}$ & CR-10 & $\mathbf{0 , 4 0}$ & CR-10 & $\mathbf{0 , 2 2}$ \\
\hline
\end{tabular}

Fuente: Elaboración propia.

Como se puede observar en las tablas anteriores, en el caso de las casas de pueblo independientes la oferta está muy distribuida por el territorio, ya que se ha pasado de un $22 \%$ entre las diez primeras en el 2000 (y un $12 \%$ entre las tres primeras) a un 19\% (y un 9\% entre las tres primeras). Destacan los municipios de la Vall de Boí (en la Alta Ribagorça), con alrededor de 50 alojamientos de este tipo, aunque ello ha representado una bajada porcentual (del 8\% en el 2000 al 2\% en el 2012); y de la Vall de Bianya (en la Garrotxa), que ha pasado de 14 a 28 (de un 2\% al 1\%).

En el caso de las casas de pueblo compartidas, la concentración ha sido algo mayor, ya que entre los diez municipios con mayor oferta de este tipo se han repartido alrededor del 25\% de estos alojamientos (y el 10\% entre los tres primeros). Sin embargo, en valores absolutos, la cantidad de estos establecimientos en cada municipio es muy baja, ya que en el 2000 ninguno sobrepasaba los cuatro, y en el 2010 y el 2012 solamente la Vall de Boí ha sobrepasado los diez.

En cuanto a las masías y masoverías, se ha producido una importante transformación en su distribución territorial, ya que se ha pasado de repartirse el $40 \%$ entre los diez municipios con más establecimientos de este tipo (y un 20\% entre los tres primeros) al 14\% (y un 5\% entre los tres primeros). Destaca la oferta en valor absoluto de los municipios de la Vall de Bianya y de Lladurs (Solsonès), que han pasado de tener cada uno menos de cuatro en el 2000 a 28 y 22 , respectivamente, en el 2012.

En el conjunto de todas las tipologías, a nivel municipal se ha pasado de una concentración geográfica del $22 \%$ entre los diez municipios con más establecimientos de turismo rural en el 2000 (y el 12\% entre los tres primeros) al 1\% (y el 5\% entre los tres primeros). 
Análogamente, en el caso de las marcas turísticas, los resultados obtenidos son los siguientes (Tablas 8 a 10):

Tabla 8. Índice de concentración relativa a nivel de marca turística (año 2012).

\begin{tabular}{|c|c|c|c|c|c|c|c|}
\hline $\begin{array}{l}\text { casa de pueblo } \\
\text { independiente }\end{array}$ & & $\begin{array}{c}\text { casa de pueblo } \\
\text { compartida }\end{array}$ & ma & a/masoveria & & total & \\
\hline Pirineus & 0,39 & Pirineus & 0,49 & Pirineus & 0,25 & Pirineus & 0,31 \\
\hline Costa Daurada & 0,16 & Costa Brava & 0,13 & Costa Brava & 0,20 & Costa Brava & 0,17 \\
\hline Costa Brava & 0,12 & Catalunya Central & 0,12 & Costa Daurada & 0,17 & Costa Daurada & 0,16 \\
\hline Terres de Lleida & 0,11 & Costa Daurada & 0,10 & Catalunya Central & 0,15 & Catalunya Central & 0,13 \\
\hline Catalunya Central & 0,10 & Terres de Lleida & 0,07 & Terres de Lleida & 0,13 & Terres de Lleida & 0,12 \\
\hline CR-3 & 0,67 & R- & 0,74 & CR-3 0,61 & & CR-3 & 0,64 \\
\hline CR-5 & 0,88 & R- & 0,91 & CR-5 0,90 & & CR-5 & 0,90 \\
\hline
\end{tabular}

Fuente: Elaboración propia.

Tabla 9. Índice de concentración relativa a nivel de marca turística (año 2006).

\begin{tabular}{|cc|cc|cc|cc|}
\hline $\begin{array}{c}\text { casa de pueblo } \\
\text { independiente }\end{array}$ & \multicolumn{2}{|c|}{$\begin{array}{c}\text { casa de pueblo } \\
\text { compartida }\end{array}$} & masía/masoveria & \multicolumn{2}{|c|}{ total } \\
\hline Pirineus & 0,39 & Pirineus & 0,55 & Pirineus & 0,37 & Pirineus & 0,41 \\
Catalunya Central & 0,17 & Costa Brava & 0,12 & Costa Brava & 0,26 & Costa Brava & 0,17 \\
Costa Brava & 0,17 & Costa Daurada & 0,10 & Catalunya Central & 0,16 & Catalunya Central & 0,15 \\
Costa Daurada & 0,09 & Terres de Lleida & 0,10 & Costa Daurada & 0,08 & Costa Daurada & 0,09 \\
Terres de Lleida & 0,08 & Val d'Aran & 0,05 & Terres de l'Ebre & 0,06 & Terres de Lleida & 0,08 \\
\hline CR-3 0,73 & & CR-3 0, & $\mathbf{7 7}$ & CR-3 & $\mathbf{0 , 7 8}$ & CR-3 & $\mathbf{0 , 7 3}$ \\
\hline CR-5 0,90 & CR-5 0, & $\mathbf{9 3}$ & CR-5 & $\mathbf{0 , 9 1}$ & CR-5 & $\mathbf{0 , 9 0}$ \\
\hline
\end{tabular}

Fuente: Elaboración propia.

Tabla 10. Índice de concentración relativa a nivel de marca turística (año 2000).

\begin{tabular}{|cc|cc|cc|cr|}
\hline $\begin{array}{c}\text { casa de pueblo } \\
\text { independiente }\end{array}$ & \multicolumn{2}{|c|}{$\begin{array}{c}\text { casa de pueblo } \\
\text { compartida }\end{array}$} & mas & ía/masoveria & \multicolumn{2}{c|}{ total } \\
\hline Pirineus & 0,48 & Pirineus & 0,38 & Pirineus & 0,65 & Pirineus & 0,51 \\
Catalunya Central & 0,15 & Costa Brava & 0,32 & Terres de Lleida & 0,09 & Costa Brava & 0,15 \\
Costa Brava & 0,13 & Catalunya Central & 0,18 & Val d'Aran & 0,08 & Catalunya Central & 0,12 \\
Terres de l'Ebre & 0,08 & Costa de Barcelona & 0,04 & Costa Brava & 0,07 & Terres de l'Ebre & 0,06 \\
Costa Daurada & 0,07 & Terres de l'Ebre & 0,04 & Terres de l'Ebre & 0,05 & Costa Daurada & 0,06 \\
\hline CR-3 0,76 & & CR-3 & $\mathbf{0 , 8 8}$ & CR-3 & $\mathbf{0 , 8 1}$ & CR-3 & $\mathbf{0 , 7 8}$ \\
\hline CR-5 0,91 & & CR-5 & $\mathbf{0 , 9 6}$ & CR-5 & $\mathbf{0 , 9 3}$ & CR-5 & $\mathbf{0 , 9 0}$ \\
\hline
\end{tabular}

Fuente: Elaboración propia.

Para las casas de pueblo independientes, cinco de las ocho marcas copan alrededor del $90 \%$ de estos establecimientos en los tres años analizados; y en tres de ellas se encuentran más de las dos terceras partes. Así, la marca Pirineus ocupa la primera posición en cada uno de los tres años, con porcentajes siempre superiores al 35\%, 
aunque con una tendencia decreciente (pasando del 48\% en el 2000 al 39\%), debido al mayor crecimiento de la oferta de estos establecimientos en muchas de las restantes marcas turísticas. Por su parte, la marca Catalunya Central, que ocupaba la segunda posición en los años 2000 y 2006 (con una oferta del $15 \%$ y del $17 \%$, respectivamente), en el 2012 ha bajado porcentualmente hasta la quinta plaza (con el $10 \%$ del total), mientras que la marca Costa Brava se ha mantenido siempre en la tercera posición en el conjunto de Cataluña, aunque pasando del 13\% en el 2000 al $12 \%$ en el 2012. La marca que más ha crecido en porcentaje en este período ha sido la Costa Daurada, que ha pasado del $7 \%$ y la quinta plaza en el 2000 al $16 \%$ y la segunda posición en el 2012.

En el caso de las casas de pueblo compartidas, los resultados obtenidos indican que en cada uno de estos años entre cinco marcas turísticas poseen más del $91 \%$ de estos alojamientos y, más concretamente, entre tres ofertan más del $74 \%$ del total, ocupando siempre las dos primeras posiciones las marcas Pirineus (con un porcentaje alrededor del 50\%) y Costa Brava (el 13\%), aunque ambas han seguido tendencias opuestas. La primera ha subido del $38 \%$ en el 2000 hasta el $49 \%-50 \%$, mientras que la segunda ha bajado del $32 \%$ en el 2000 hasta el 12\%-13\%. Las restantes marcas han ido fluctuando sus posiciones en esta clasificación. Unas se han mantenido (Costa Daurada y Terres de Lleida) y otras han descendido (Costa de Barcelona o Val d'Aran), aunque siempre con una oferta nunca superior al 5\% del total. Destaca el caso de la marca Catalunya Central, que ocupaba la tercera posición en el 2000 (con una oferta del 18\%), desapareciendo de las cinco primeras plazas en el 2006 y volviendo a la tercera posición en el 2012 (con el 12\%).

Finalmente, con relación a las masías y masoverías, aunque entre cinco marcas turísticas se reparten más del $90 \%$ de la oferta, su distribución se ha extendido por todo el territorio catalán, de modo que entre las tres primeras se ha pasado del $81 \%$ (año 2000) al 61\% (año 2012). La marca Pirineus ha mantenido su posición privilegiada, aunque ha bajado del $65 \%$ al $25 \%$, mientras que muchas de las restantes marcas han ido aumentando su cuota, destacando la Costa Brava (pasando del 7\% al $20 \%$ ), Costa Daurada (de menos del $5 \%$ al $17 \%$ ) y Catalunya Central (de menos del $5 \%$ al $15 \%)$.

\section{3. ÍNDICE DE CONCENTRACIÓN GEOGRÁFICA DE ELLISON-GLAESER}

Los resultados de los cálculos del Índice de Ellison-Glaeser para cada una de las tipologías de establecimientos de turismo rural industrial, en cada uno de los tres años aquí considerados, son los que se presentan en la tabla siguiente (Tabla 11). 
Tabla 11. Índice de Ellison-Glaeser

\begin{tabular}{|l|c|c|c|c|c|}
\hline \multicolumn{1}{|l|}{ índice de Ellison-Glaeser } & $\begin{array}{c}\text { casa de pueblo } \\
\text { compartida }\end{array}$ & $\begin{array}{c}\text { casa de pueblo } \\
\text { independiente }\end{array}$ & $\begin{array}{c}\text { masía/ } \\
\text { masovería total }\end{array}$ \\
\hline comarcas & 2012 & 0,0407 & 0,0176 & 0,0084 & 0,0396 \\
& 2006 & 0,0571 & 0,0028 & 0,0079 & $-0,0006$ \\
& 2000 & 0,0262 & 0,0050 & 0,0468 & $-0,0016$ \\
\hline municipios con ETR & & & & & \\
& 2012 & 0,2914 & 0,0182 & 0,0112 & 0,0179 \\
& 2006 & 0,0518 & 0,0014 & 0,0084 & $-0,0006$ \\
& 2000 & 0,2787 & 0,0519 & 0,1610 & $-0,0016$ \\
\hline marcas turísticas & & & & & \\
& 2012 & 0,2508 & 0,0047 & 0,0039 & $-0,0006$ \\
& 2006 & 0,2269 & 0,0023 & 0,2847 & $-0,0006$ \\
& 2000 & 0,0389 & 0,0066 & 0,0718 & $-0,0016$ \\
\hline
\end{tabular}

Fuente: Elaboración propia.

Como se aprecia en la tabla anterior, los resultados obtenidos presentan grandes similitudes, tanto en función del área territorial analizada (comarca, municipio, marca turística) como en su evolución temporal (años 2000-2006-2012). Así, a nivel comarcal, en el caso de las casas de pueblo compartidas se han obtenido unos valores entre 0,0262 y 0,0571 , lo que muestra una concentración moderada, aumentando un $55 \%$ en el período (con una subida porcentual del $117 \%$ en los primeros seis años y una bajada del $28 \%$ en los segundos seis); mientras que en las casas de pueblo independientes y en las masías y masoverías la concentración era muy baja, con valores entre 0,0028 y 0,0468 , aunque las primeras han experimentado en este tiempo un crecimiento del $252 \%$ (especialmente en estos últimos seis años, en que éste ha sido del 528\%) y las segundas un decrecimiento del $82 \%$ (especialmente en los primeros seis años). Todo ello demuestra que durante este período, a nivel comarcal, la concentración de estos establecimientos sigue las mismas pautas que el conjunto de Cataluña, sin presentarse claras fuerzas de aglomeración.

A nivel municipal, la concentración varía según el tipo de alojamiento, con valores entre 0,2914 y 0,0518 (alta concentración) para las casas de pueblo compartidas; entre 0,0014 (muy baja) y 0,0519 (alta) para las casas de pueblo independientes; y entre 0,0084 (baja) y 0,1610 (alta concentración) para las masías y masoverías. En los tres casos, durante los primeros seis años el índice ha disminuido cerca del $100 \%$ y en los segundos seis ha subido significativamente, aunque sin llegar a alcanzar los valores iniciales, excepto en el caso de las casas de pueblo compartidas.

A nivel de marcas turísticas, la concentración es muy variable, según la tipología del establecimiento y el año analizado, ya que todos los valores oscilan entre 0,0023 (muy baja) y 0,2847 (alta). Así, para las casas de pueblo independientes siempre se ha 
obtenido un valor muy cercano a cero (sin concentración geográfica); mientras que para las casa de pueblo compartidas la concentración ha pasado de media a alta (de 0,0389 a 0,2508 ), con un fuerte aumento en este período (el 544\%), siendo el $483 \%$ en los primeros seis años y manteniendo una estabilización en los otros seis (con un aumento solamente del 10\%); y para las masías y masoverías ha ocurrido el fenómeno contrario, pasando de una alta concentración $(0,0718)$ a otra baja $(0,0039)$, de modo que el aumento de los primeros seis años (el 296\%) se ha visto contrarrestado parcialmente con una bajada posterior del $98 \%$.

\section{CONCLUSIONES}

La aplicación de la metodología expuesta en este trabajo, basada en los índices de Gini, de Concentración Relativa y Ellison-Glaeser, ha permitido analizar la concentración de los establecimientos de turismo rural en Cataluña, en los años 2000, 2006 y 2012, utilizando como unidad territorial el municipio, la comarca y la marca turística.

La utilización de estas diferentes escalas ha puesto de manifiesto que, según los valores del índice de Gini, los niveles de concentración de las diferentes tipologías de estos alojamientos son mayores en los niveles superiores al municipal (los casos de las comarcas y de las marcas turísticas), teniendo en cuenta que la mayor concentración territorial se produce con las masía y masoverías, seguida por las casa de pueblo compartidas y, finalmente, por las casa de pueblo independientes.

En cuanto a su evolución en estos tres años, la concentración de las masías y masoverías y de las casas de pueblo compartidas ha aumentado considerablemente cuanto mayor era la unidad de análisis, mientras que ha disminuido a nivel municipal. Así pues, la oferta de estos tipos de alojamientos se ha distribuido por más municipios pero dentro de las mismas comarcas, aunque en diferentes marcas turísticas. En el caso de las casas de pueblo independientes casi no ha habido evolución, manteniéndose bastante estabilizados los niveles de concentración en todas las unidades territoriales analizadas. En el conjunto de las tres tipologías, aunque el grado de concentración se ha mantenido estable a nivel municipal, en el nivel comarcal ha aumentado y en el de las marcas turísticas ha disminuido.

Mediante el coeficiente de Ellison-Glaeser se han calculado los niveles de aglomeración de estos alojamientos y se ha visto que son mayores en el caso de las casas de pueblo compartidas y casi inapreciables en el resto de tipologías. Sin embargo, su evolución en este período ha puesto de manifiesto una gran variabilidad en todos los tipos de alojamientos, pasando de una cierta aglomeración a su inexistencia, y viceversa, sin un patrón que justifique estas oscilaciones.

Estos resultados indican algunas desviaciones no insignificantes según la agregación territorial analizada, lo que puede interpretarse como una evidencia de que los efectos que conducen a la aglomeración no acaban en el límite administrativo del municipio. La ausencia de estos efectos de desbordamiento entre unidades geográficas, principalmente entre comarcas, podría generar una distribución aleatoria 
del número de plazas ofertadas en cada tipología. Así, encontramos comarcas con muchas plazas de turismo rural de una determinada tipología al lado de otras donde estos alojamientos tienen muy poca presencia. En cambio, si las fuerzas que conducen a la aglomeración superan los límites administrativos comarcales, como es el caso de las marcas turísticas, se puede observar una correlación más positiva del número de plazas entre las comarcas más próximas geográficamente.

Finalmente, mediante el índice de Concentración Relativa se ha puesto de manifiesto que los establecimientos de turismo rural están repartidos de manera poco uniforme por toda Cataluña, concentrándose especialmente en las comarcas más septentrionales (Pirineos y Pre-Pirineos), exceptuando los casos del Valle de Arán y de la Cerdanya, donde predomina el turismo residencial. Sin embargo su evolución en estos años no presenta ninguna tendencia clara (Figura 4).

Figura 4. Mapa con las comarcas con mayor oferta de establecimientos de turismo rural en Cataluña según su tipología (años 2000, 2006 y 2012).

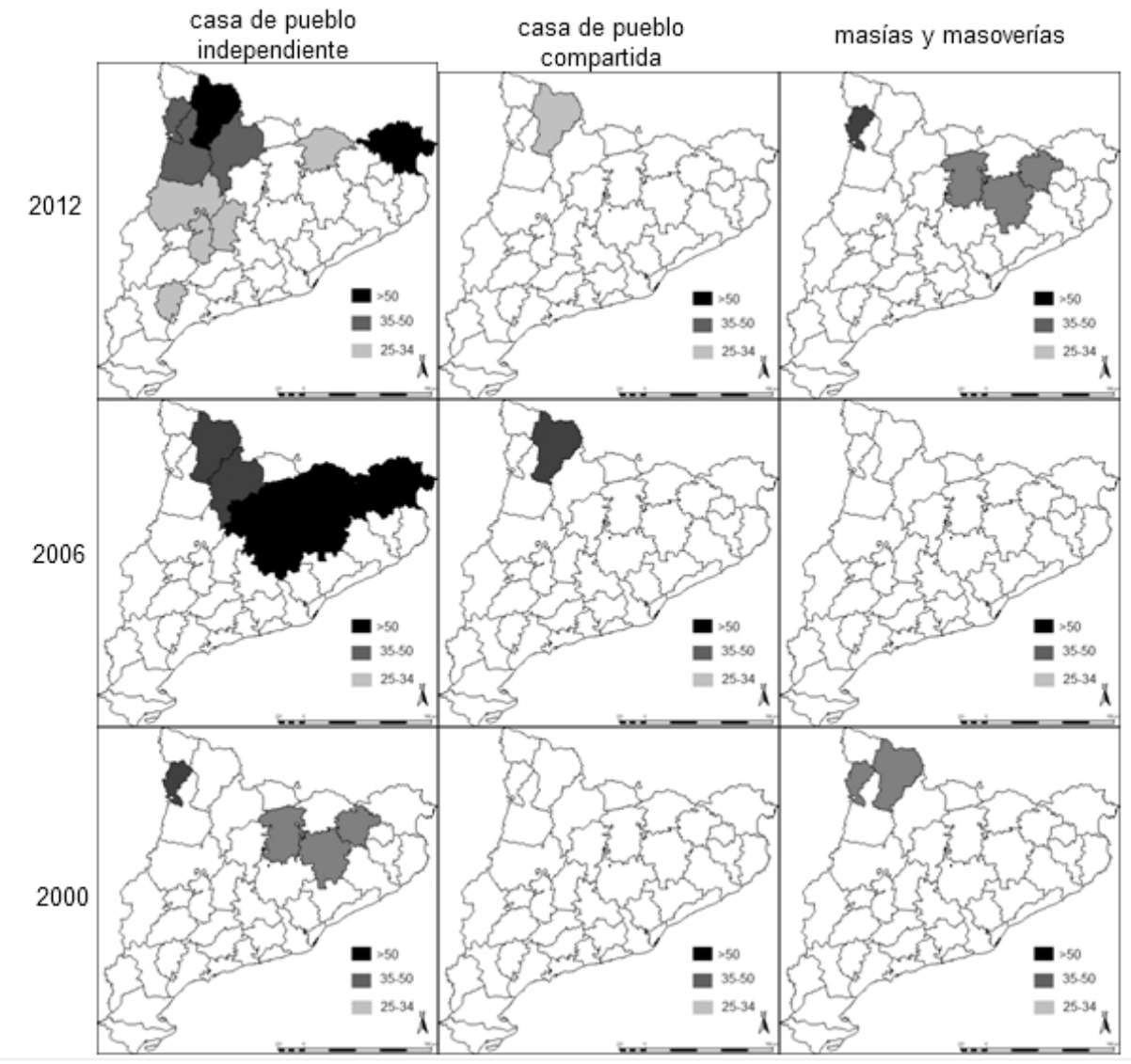

Fuente: Elaboración propia con Miramon. 
Estos resultados, según los criterios comúnmente aceptados (Novelli et al., 2006; Viladecans y Jofre, 2006), muestran una concentración moderada. Por ello, no podemos validar la hipótesis de que las economías de aglomeración juegan un papel activo en la localización de los establecimientos de turismo rural, pero tampoco podemos afirmar que no juegan ningún papel. Sin embargo, sí que parece claro que es necesaria una política de promoción y desarrollo de este tipo de turismo a nivel comarcal, con la participación de los organismos públicos y de los establecimientos privados.

\section{BIBLIOGRAFÍA}

ALAÑON, A., ARAUZO, J.M. Y MYRO, R. (2007): Accesibility, Agglomeration and Location. En Arauzo, J.M. y Manjón, M. Eeds.) Entrepreneurship, Industrial Location and Economic Growth. Chentelham, Edward Elgar.

ÁVILA, R. Y BARRADO, D. (2005): Nuevas tendencias en el desarrollo de destinos turísticos: marcos conceptuales y operativos para su planificación y gestión. Cuadernos de Turismo, 15, 27-43.

AUDRETSCH D.B. Y FELDMAN, M.P. (1996): R\&D spillovers and the geography of innovation and production. American Economic Review, 86, 630-640.

BOIX, R. Y GALLETTO, V. (2009): Innovation and industrial districts: a first approach to the measurement and determinants of the I-district effect. Regional Studies, 43 (9), 1117-1133.

BRENNER, T. (2006): Identification of Local Industrial Clusters in Germany. Regional Studies, 40, 1-14.

CÁNOVES, G., VILlARINO, M., HERRERA, L., CUESTA, L. (2004): Turismo rural en Cataluña y Galicia: algunos problemas sin resolver. Cuadernos Geográficos, 34, 111-128.

CÀNOVES, G., HERRERA, L. Y CUESTA, L. (2006): El turismo rural en Cataluña: una apuesta medioambiental y de calidad. Barcelona, Fundación Abertis.

CAPONE, F. Y BOIX, R. (2008): Sources of growth and competitiveness of local tourist production systems: an application to Italy (1991-2001). The Annals of Regional Science, 42 (1), 209-224.

COSTA, M.T. (1992): Cambios en la organización industrial: Cooperación local y competitividad internacional. Panorama general. Economía industrial, 286, 19-36.

DEVEREUX, M.P., GRIFFITH, R. Y SIMPSON, H. (2004): The Geographic Distribution of Production Activity in the UK. Regional Science and Urban Economics, 35 (5), 533-564.

DURANTON, G. Y OVERMAN, H. (2005): Testing for localization using microgeographic data. Review of Economic Studies, 72, 1077-1106.

ELLISON, G. Y GLAESER, E.L. (1997): Geographic Concentration in U.S. Manufacturing Industries: A Dartboard Approach. Journal of Political Economy, 105 (5), 889-927. 
ELLISON, G. Y GLAESER, E.L. (1999): The geographic concentration of industry: Does natural advantages explain agglomeration?. American Economic Review. Papers and Procedings, 89 (2), 311-316.

GINER, J.M. Y SANTAMARÍA, M.J. (2002): Territorial systems of small firms in Spain: an analysis of productive and organizational characteristics in industrial districts. Entrepreneurship \& Regional Development, 14, 211-228.

GINI, C. (1912): Variabilita e mutabilita: Contributi allo studio della destribuzioni e relazioni stadistiche. Studi Economico Giuridice dell' Universita di Calgari, 3, 1158.

GREENE, W.H. (2003): Econometric Analysis. New Jersey, Prentice Hall.

GUIMARAES, P., FIGUEREIDO, O. Y WOODWARD, D. (2000): Agglomeration and the Location of Foreign Direct Investment in Portugal. Journal of Urban Economics, 47, 115-135.

KRUGMAN, P. (1991): Geography and Trade. Cambridge, MIT Press.

LUCIO, J.J. DE (1998): Un análisis global, regional y sectorial de los efectos externos de conocimiento. Working Paper, 98-03, FEDEA.

MILLÁN, M. (2008): La planificación turística y capital territorial. Un enfoque geográfico. En Ivars y Vera J.F. (Eds.) Espacios turísticos. Mercantilización, paisaje e identidad, 127-140.

NOVELLI, M., SCHMITZ, B. Y SPENCER, T. (2006): Networks, cluster and innovation in tourism:A UK experience. Tourism Management, 27, 1141-1152.

O'DONOGHUE, D. Y GLEAVE, B. (2004): A note on methods for measuring industrial agglomeration. Regional Studies, 38 (4), 419-427.

OTTAVIANO G. Y THISSE J.F. (2005): New economic geography: what about the N?. Environment and Planning, 37 (10), 1707-1725.

PULIDO, J. I. (2008): El turismo rural: Estructura económica y configuración territorial en España. Madrid, Síntesis.

ROSENTHAL, S. Y STRANGE, W.C. (2003): Geography, Industrial Organisation and Agglomeration. The Review of Economics and Statistics, 50, 191-229.

SANTAMARÍA, M.J., GINER, J.M. Y FUSTER, A. (2004): Identification of the local productive systems in Spain: A new approach. 44Th Congress of ERSA, Porto (Portugal).

SOLER, V. (2006): Nuevas técnicas para la medición del efecto distrito en las aglomeraciones industriales. Economía Industrial, 359, 81-87.

STRANGE, W.C. (2005): Agglomeration. En Eatwell, J., Milgate, M y Newman, P. (Eds.). New Palgrave Dictionary of Economics. New York, Palgrave MacMillan.

TICKELL, A. (2002): Geography of Services: Progress in the Geography of Services III-Time to Move on?. Progress in Human Geography, 26, 791-801.

VANHOVE, N (2002): Tourism Policy -Between Competitiveness and Sustainability: The Case of Bruges. Tourism Review, 57, $\mathrm{n}^{\circ} 3$.

VILADECANS, E. (2000): Economies externes i concentració de les activitats manufactureres: Una anàlisi del municipis espanyols. Revista Econòmica de Catalunya, 39, 53-62. 
VILADECANS, E. (2004): Agglomeration economies and industrial location: citylevel evidence. Journal of Economic Geography, 4/5, 565-482.

VILADECANS, E. Y JOFRE, J. (2006): La localització geográfica de la indústria a Catalunya: El paper de els economies d'aglomeració. Barcelona, Papers d'Economia Industrial. Departament de Treball e Indústria. Generalitat de Catalunya, $202 \mathrm{p}$.

YBARRA, J.A. (1991): Determinación cuantitativa de distritos industriales: la experiencia del País Valenciano. Estudios Territoriales, 37, 53-67. 